\title{
On Hurwitzian and Tasoev's continued fractions
}

\author{
by \\ TAKaO Komatsu (Mie) \\ Dedicated to Professor Iekata Shiokawa \\ on the occasion of his 60th birthday
}

1. Introduction. Hurwitzian continued fraction expansions have the form

$$
\begin{aligned}
& {\left[c_{0} ; c_{1}, \ldots, c_{n}, \overline{Q_{1}(k), \ldots, Q_{p}(k)}\right]_{k=1}^{\infty}} \\
& \quad=\left[c_{0} ; c_{1}, \ldots, c_{n}, Q_{1}(1), \ldots, Q_{p}(1), Q_{1}(2), \ldots, Q_{p}(2), Q_{1}(3), \ldots\right]
\end{aligned}
$$

where $c_{0}$ is an integer, $c_{1}, \ldots, c_{n}$ are positive integers, $Q_{1}, \ldots, Q_{p}$ are polynomials with rational coefficients which take positive integral values for $k=1,2, \ldots$ and at least one of the polynomials is not constant (see e.g. [1], [8], [9]). Well-known examples are

$$
\begin{aligned}
e & =[2 ; 1,2,1,1,4,1,1,6,1, \ldots]=[2 ; \overline{1,2 k, 1}]_{k=1}^{\infty}, \\
\tanh 1 & =\frac{e^{2}-1}{e^{2}+1}=[0 ; 1,3,5,7, \ldots]=[0 ; \overline{2 k-1}]_{k=1}^{\infty}, \\
\tan 1 & =[1 ; 1,1,3,1,5,1, \ldots]=[1 ; \overline{2 k-1,1}]_{k=1}^{\infty} .
\end{aligned}
$$

The forms

$$
\frac{\alpha e^{2 / g}+\beta}{\gamma e^{2 / g}+\delta} \quad \text { with } \alpha, \beta, \gamma, \delta, g \in \mathbb{Z}, g \neq 0, \alpha \delta-\beta \gamma \neq 0
$$

are called Hurwitz numbers ([4], [5]). But the general relation between Hurwitz numbers and Hurwitzian continued fraction expansions has not been discovered yet (see [15, p. 129], e.g.).

Tasoev [13], [14] proposed a new continued fraction which is like Hurwitzian but $Q_{j}(k)$ includes exponentials in $k$ instead of polynomials. The author [6] obtained the closed form of $[0 ; \underbrace{\overline{a^{k}, \ldots, a^{k}}}_{m}]_{k=1}^{\infty}$ by using the method

2000 Mathematics Subject Classification: Primary 11A55, 11J70. 
of Wall [16]. When $m=1$ or 2 , its closed form is simple with the fraction of two infinite sums. In this paper some more general cases are treated. Furthermore, in each case a continued fraction expansion can be obtained, by using the negative continued fraction.

The same technique can be applied to Hurwitzian continued fraction expansions. Once we get a continued fraction whose partial quotients consist of arithmetic progressions (we call it of tanh type), we easily deduce its counterpart (called tan type) with the negative continued fraction instead of the simple continued fraction. More extended cases will also be mentioned.

2. The simple and negative continued fraction expansions. As usual, $\alpha=\left[a_{0}, a_{1}, a_{2}, \ldots\right]$ denotes the simple continued fraction expansion of $\alpha$, where

$$
\begin{aligned}
& \alpha=a_{0}+\theta_{0}, \quad a_{0}=\lfloor\theta\rfloor, \\
& 1 / \theta_{n-1}=a_{n}+\theta_{n}, \quad a_{n}=\left\lfloor 1 / \theta_{n-1}\right\rfloor \quad(n \geq 1) .
\end{aligned}
$$

The $n$th convergent $p_{n} / q_{n}=\left[a_{0} ; a_{1}, \ldots, a_{n}\right]$ of $\alpha$ is given by the recurrence relations

$$
\begin{array}{cccc}
p_{n}=a_{n} p_{n-1}+p_{n-2} & (n \geq 0), & p_{-1}=1, & p_{-2}=0 \\
q_{n}=a_{n} q_{n-1}+q_{n-2} & (n \geq 0), & q_{-1}=0, & q_{-2}=1 .
\end{array}
$$

We now introduce the negative continued fraction expansion. We use the definition and notation in [10] with a change of the position of the minus sign, because it is more convenient when $0<\alpha<1$. The usual definition can be found in [3]. The negative expansion of a real $\alpha$ is denoted by

$$
\alpha={ }^{-}\left[0 ; a_{1}, a_{2}, a_{3}, \ldots\right]=\frac{1}{a_{1}-\frac{1}{a_{2}-\frac{1}{a_{3}-\ldots}}}
$$

where the integers $a_{i} \geq 2$ are generated by ceiling functions rather than floor functions in the continued fraction algorithm:

$$
\begin{aligned}
\theta_{0} & =\{\alpha\}, \\
1 / \theta_{n-1} & =a_{n}-\theta_{n}, \quad a_{n}=\left\lceil 1 / \theta_{n-1}\right\rceil \quad(n \geq 1)
\end{aligned}
$$

with corresponding convergents $p_{n} / q_{n}={ }^{-}\left[0 ; a_{1}, \ldots, a_{n}\right]$ given by

$$
\begin{aligned}
& p_{n}=a_{n} p_{n-1}-p_{n-2} \quad(n \geq 1), \quad p_{0}=0, \quad p_{-1}=-1, \\
& q_{n}=a_{n} q_{n-1}-q_{n-2} \quad(n \geq 1), \quad q_{0}=1, \quad q_{-1}=0 .
\end{aligned}
$$

A simple continued fraction expansion can be transformed into a negative continued fraction expansion by the following rule. 
LEMMA 1 ([10]).

$$
\begin{aligned}
& {\left[0 ; a_{1}, a_{2}, a_{3}, a_{4}, a_{5}, a_{6}, a_{7}, \ldots\right]} \\
& \quad={ }^{-}[0 ; a_{1}+1, \underbrace{2, \ldots, 2}_{a_{2}-1}, a_{3}+2, \underbrace{2, \ldots, 2}_{a_{4}-1}, a_{5}+2, \underbrace{2, \ldots, 2}_{a_{6}-1}, a_{7}+2, \ldots] .
\end{aligned}
$$

Proof. We refer to [11, p. 227]. Since

$$
\begin{aligned}
{[\ldots, a, b, c, d, \ldots] } & =[\ldots, a, 0,-1,1,-1,0,-b,-c, \ldots] \\
& =[\ldots, a-1,1,-b-1,-c, \ldots]
\end{aligned}
$$

or

$$
\begin{aligned}
{[\ldots, a, b, c, d, \ldots] } & =[\ldots, a, 0,1,-1,1,0,-b,-c, \ldots] \\
& =[\ldots, a+1,-1,-b+1,-c, \ldots]
\end{aligned}
$$

we have

$$
\begin{aligned}
& {[\ldots, a, b, c, d, e, \ldots]=[\ldots, a+1,-1,-b+1,-c,-d,-e, \ldots]} \\
& \quad=[\ldots, a+1,-2,1, b-2, c, d, e, \ldots] \\
& \quad=[\ldots, a+1,-2,2,-1,-b+3,-c,-d,-e, \ldots] \\
& \quad=\ldots \\
& \quad=[\ldots, a+1, \underbrace{-2,2, \ldots,(-2)^{b-1}}_{b-1},(-1)^{b}(c+1),(-1)^{b} d,(-1)^{b} e, \ldots] .
\end{aligned}
$$

\section{Tasoev's continued fractions}

THEOREM 1. Let $a$ be an integer and $u$ a rational with $a>1$ and $u a \in$ $\mathbb{Z}^{+}$. Then

$$
\left[0 ; \overline{u a^{k}}\right]_{k=1}^{\infty}=\frac{\sum_{s=0}^{\infty} u^{-2 s-1} a^{-(s+1)^{2}} \prod_{i=1}^{s}\left(a^{2 i}-1\right)^{-1}}{\sum_{s=0}^{\infty} u^{-2 s} a^{-s^{2}} \prod_{i=1}^{s}\left(a^{2 i}-1\right)^{-1}} .
$$

REMARK. If $u=1$, then we have the first relation of the main theorem in $[6]$.

Proof of Theorem 1. Let the power series

$$
f_{n}(z)=r_{n, 0}+r_{n, 1} z+r_{n, 2} z^{2}+\ldots
$$

satisfy the relation

$$
f_{n}(z)=u a^{n+1} f_{n+1}(z)+z f_{n+2}(z)
$$

for $n=0,1,2, \ldots$ Then from

$$
\frac{f_{n}(z)}{f_{n+1}(z)}=u a^{n+1}+\frac{z}{\frac{f_{n+1}(z)}{f_{n+2}(z)}}
$$


we obtain the desired continued fraction expansion as

$$
\frac{f_{1}(1)}{f_{0}(1)}=\left[0 ; u a, u a^{2}, u a^{3}, \ldots\right]
$$

By comparing the constant and the coefficient of $z^{s}(s \geq 1), r_{n, l}$ must satisfy the recurrence relations

$$
\begin{aligned}
& r_{n, 0}=u a^{n+1} r_{n+1,0}, \\
& r_{n, s}=u a^{n+1} r_{n+1, s}+r_{n+2, s-1} .
\end{aligned}
$$

By the first relation we have $r_{0,0}=u^{n} a^{n(n+1) / 2} r_{n, 0}$. We can assume that $r_{0,0}=1$. Otherwise, any of $r_{n, s}$ will be multiplied by $r_{0,0}$ and finally $r_{0,0}$ will be cancelled in $f_{1}(1) / f_{0}(1)$. Hence,

$$
r_{n, 0}=u^{-n} a^{-n(n+1) / 2} .
$$

By the second relation we have

$$
r_{0, s}=u^{n} a^{n(n+1) / 2} r_{n, s}+\sum_{k=1}^{n} u^{k-1} a^{(k-1) k / 2} r_{k+1, s-1} .
$$

By induction we shall show

$$
r_{n, s}=u^{-n-2 s} a^{-n(n+1) / 2-2 s n-s^{2}} \prod_{i=1}^{s}\left(a^{2 i}-1\right)^{-1} .
$$

For $s=1$ by (1) and (2) we have

$$
\begin{aligned}
r_{0,1} & =u^{n} a^{n(n+1) / 2} r_{n, 1}+\sum_{k=1}^{n} u^{k-1} a^{(k-1) k / 2} u^{-k-1} a^{-(k+1)(k+2) / 2} \\
& =u^{n} a^{n(n+1) / 2} r_{n, 1}+u^{-2} \sum_{k=1}^{n} a^{-2 k-1} \\
& =u^{n} a^{n(n+1) / 2} r_{n, 1}+u^{-2} a^{-1}\left(1-a^{-2 n}\right)\left(a^{2}-1\right)^{-1} .
\end{aligned}
$$

Separating the terms including $n$ from the constant terms without $n$, we have

$$
r_{n, 1}=u^{-n-2} a^{-n(n+1) / 2-2 n-1}\left(a^{2}-1\right)^{-1} .
$$

Assume that the assertion is valid for $s-1$ and $s$. Then by (2) we have

$$
\begin{aligned}
r_{0, s+1} & \\
= & u^{n} a^{n(n+1) / 2} r_{n, s+1} \\
& +\sum_{k=1}^{n} u^{k-1} a^{(k-1) k / 2} u^{-k-1-2 s} a^{-(k+1)(k+2) / 2-2 s(k+1)-s^{2}} \prod_{i=1}^{s}\left(a^{2 i}-1\right)^{-1}
\end{aligned}
$$




$$
\begin{aligned}
& =u^{n} a^{n(n+1) / 2} r_{n, s+1}+u^{-2 s-2} \sum_{k=1}^{n} a^{-2(s+1) k-(s+1)^{2}} \prod_{i=1}^{s}\left(a^{2 i}-1\right)^{-1} \\
& =u^{n} a^{n(n+1) / 2} r_{n, s+1}+u^{-2(s+1)} a^{-(s+1)^{2}}\left(1-a^{-2(s+1) n}\right) \prod_{i=1}^{s+1}\left(a^{2 i}-1\right)^{-1} .
\end{aligned}
$$

Separating the terms including $n$ from the constant terms without $n$, we have

$$
r_{n, s+1}=u^{-n-2(s+1)} a^{-n(n+1) / 2-2(s+1) n-(s+1)^{2}} \prod_{i=1}^{s+1}\left(a^{2 i}-1\right)^{-1} .
$$

Therefore, we obtain the desired form

$$
\left[0 ; \overline{u a^{k}}\right]_{k=1}^{\infty}=\frac{f_{1}(1)}{f_{0}(1)}=\frac{\sum_{s=0}^{\infty} r_{1, s}}{\sum_{s=0}^{\infty} r_{0, s}} .
$$

THEOREM 2. Let $a$ be an integer and $u$ a rational with $a>1$ and $u a \in$ $\mathbb{Z}^{+}$. Then

$$
\left[0 ; u a-1, \overline{1, u a^{k+1}-2}\right]_{k=1}^{\infty}=\frac{\sum_{s=0}^{\infty}(-1)^{s} u^{-2 s-1} a^{-(s+1)^{2}} \prod_{i=1}^{s}\left(a^{2 i}-1\right)^{-1}}{\sum_{s=0}^{\infty}(-1)^{s} u^{-2 s} a^{-s^{2}} \prod_{i=1}^{s}\left(a^{2 i}-1\right)^{-1}} .
$$

Proof. Let the power series $f_{n}(z)=r_{n, 0}+r_{n, 1} z+r_{n, 2} z^{2}+\ldots$ satisfy the relation

$$
f_{n}(z)=u a^{n+1} f_{n+1}(z)-z f_{n+2}(z)
$$

instead of $f_{n}(z)=u a^{n+1} f_{n+1}(z)+z f_{n+2}(z)$ in Theorem 1 . Then $r_{n, l}$ satisfies the recurrence relations

$$
\begin{aligned}
& r_{n, 0}=u a^{n+1} r_{n+1,0}, \\
& r_{n, s}=u a^{n+1} r_{n+1, s}-r_{n+2, s-1} .
\end{aligned}
$$

Thus, in a similar manner to the proof of Theorem 1, we can have

$$
r_{n, s}=(-1)^{s} u^{-n-2 s} a^{-n(n+1) / 2-2 s n-s^{2}} \prod_{i=1}^{s}\left(a^{2 i}-1\right)^{-1} .
$$

Therefore, from

$$
\frac{f_{1}(z)}{f_{0}(z)}=\frac{z}{u a-\frac{z}{u a^{2}-\frac{z}{u a^{3}-\ldots}}}
$$

and Lemma 1 we obtain

$$
\left[0 ; u a-1, \overline{1, u a^{k+1}-2}\right]_{k=1}^{\infty}=-\left[0 ; \overline{u a^{k}}\right]_{k=1}^{\infty}=\frac{f_{1}(1)}{f_{0}(1)}=\frac{\sum_{s=0}^{\infty} r_{1, s}}{\sum_{s=0}^{\infty} r_{0, s}}
$$


THEOREM 3. Let $a$ and $b$ be integers and $u$ and $v$ be rationals with $a, b>1$ and $u a, v b \in \mathbb{Z}^{+}$. Then

$$
\left[0 ; \overline{u a^{k}, v b^{k}}\right]_{k=1}^{\infty}=\frac{\sum_{s=0}^{\infty} r_{1, s}}{\sum_{s=0}^{\infty} r_{0, s}}
$$

where for $s=0,1, \ldots$,

$$
\begin{aligned}
r_{0,2 s} & =u^{-2 s} v^{-2 s} R_{0,2 s} a^{-s(s+1)} b^{-s^{2}} \prod_{i=1}^{2 s}\left(a^{i} b^{i}-1\right)^{-1}, \\
r_{0,2 s+1} & =u^{-2 s-1} v^{-2 s-1} R_{0,2 s+1} a^{-(s+1)^{2}} b^{-s(s+1)} \prod_{i=1}^{2 s+1}\left(a^{i} b^{i}-1\right)^{-1}, \\
r_{1,2 s} & =u^{-2 s-1} v^{-2 s} R_{1,2 s} a^{-(s+1)^{2}} b^{-s(s+1)} \prod_{i=1}^{2 s}\left(a^{i} b^{i}-1\right)^{-1}, \\
r_{1,2 s+1} & =u^{-2 s-2} v^{-2 s-1} R_{1,2 s+1} a^{-(s+1)(s+2)} b^{-(s+1)^{2}} \prod_{i=1}^{2 s+1}\left(a^{i} b^{i}-1\right)^{-1},
\end{aligned}
$$

with $R_{0,0}=R_{1,0}=1$ and for $s=0,1, \ldots$,

$$
\begin{aligned}
R_{0,2 s} & =a^{s} b^{s} R_{0,2 s-1}+R_{1,2 s-1}, \\
R_{0,2 s+1} & =a^{s+1} b^{s} R_{0,2 s}+R_{1,2 s}, \\
R_{1,2 s} & =R_{0,2 s-1}+a^{s} b^{s} R_{1,2 s-1}, \\
R_{1,2 s+1} & =R_{0,2 s}+a^{s} b^{s+1} R_{1,2 s} .
\end{aligned}
$$

Proof. Consider the power series $f_{n}(z)=r_{n, 0}+r_{n, 1} z+r_{n, 2} z^{2}+\ldots$, satisfying

$$
\begin{aligned}
f_{2 n}(z) & =u a^{n+1} f_{2 n+1}(z)+z f_{2 n+2}(z), \\
f_{2 n+1}(z) & =v b^{n+1} f_{2 n+2}(z)+z f_{2 n+3}(z) .
\end{aligned}
$$

Thus, $f_{1}(1) / f_{0}(1)=\left[0 ; \overline{u a^{k}, v b^{k}}\right]_{k=1}^{\infty}$. By comparing the constant terms we have

$$
\begin{aligned}
r_{2 n, 0} & =u a^{n+1} r_{2 n+1,0}, \\
r_{2 n+1,0} & =v b^{n+1} r_{2 n+2,0},
\end{aligned}
$$

yielding

$$
\begin{aligned}
r_{2 n, 0} & =u^{-n} v^{-n} a^{-n(n+1) / 2} b^{-n(n+1) / 2}, \\
r_{2 n+1,0} & =u^{-n-1} v^{-n} a^{-(n+1)(n+2) / 2} b^{-n(n+1) / 2} .
\end{aligned}
$$

By comparing the coefficients of $z^{s}$ we have

$$
\begin{aligned}
r_{2 n, s} & =u a^{n+1} r_{2 n+1, s}+r_{2 n+2, s-1}, \\
r_{2 n+1, s} & =v b^{n+1} r_{2 n+2, s}+r_{2 n+3, s-1},
\end{aligned}
$$


yielding

$$
\begin{aligned}
r_{0, s}= & u^{n} v^{n} a^{n(n+1) / 2} b^{n(n+1) / 2} r_{2 n, s} \\
& +\sum_{k=1}^{n}\left(u^{k} v^{k-1} a^{k(k+1) / 2} b^{(k-1) k / 2} r_{2 k+1, s-1}\right. \\
& \left.+u^{k-1} v^{k-1} a^{(k-1) k / 2} b^{(k-1) k / 2} r_{2 k, s-1}\right) .
\end{aligned}
$$

When $s=1$, we get

$$
\begin{aligned}
r_{0,1}= & u^{n} v^{n} a^{n(n+1) / 2} b^{n(n+1) / 2} r_{2 n, 1} \\
& +\sum_{k=1}^{n}\left(u^{k} v^{k-1} a^{k(k+1) / 2} b^{(k-1) k / 2} u^{-k-1} v^{-k} a^{-(k+1)(k+2) / 2} b^{-k(k+1) / 2}\right. \\
& \left.+u^{k-1} v^{k-1} a^{(k-1) k / 2} b^{(k-1) k / 2} u^{-k} v^{-k} a^{-k(k+1) / 2} b^{-k(k+1) / 2}\right) \\
= & u^{n} v^{n} a^{n(n+1) / 2} b^{n(n+1) / 2} r_{2 n, 1}+u^{-1} v^{-1} \sum_{k=1}^{n}\left(a^{-k-1} b^{-k}+a^{-k} b^{-k}\right) \\
= & u^{n} v^{n} a^{n(n+1) / 2} b^{n(n+1) / 2} r_{2 n, 1}+u^{-1} v^{-1} \cdot \frac{a+1}{a(a b-1)}\left(1-a^{-n} b^{-n}\right) .
\end{aligned}
$$

Separating the terms including $n$ from those without $n$ we obtain

$$
r_{2 n, 1}=u^{-n-1} v^{-n-1} R_{0,1} a^{-n(n+1) / 2-n-1} b^{-n(n+1) / 2-n}(a b-1)^{-1},
$$

where $R_{0,1}=a+1$. Then

$$
\begin{aligned}
r_{2 n+1,1} & =u^{-1} a^{-(n+1)}\left(r_{2 n, 1}-r_{2 n+2,0}\right) \\
= & u^{-1} a^{-(n+1)}\left(u^{-n-1} v^{-n-1} R_{0,1} a^{-n(n+1) / 2-n-1} b^{-n(n+1) / 2-n}(a b-1)^{-1}\right. \\
& \left.\quad-u^{-n-1} v^{-n-1} a^{-(n+1)(n+2) / 2} b^{-(n+1)(n+2) / 2}\right) \\
= & u^{-n-2} v^{-n-1} R_{1,1} a^{-n(n+1) / 2-2 n-2} b^{-n(n+1) / 2-n-1}(a b-1)^{-1},
\end{aligned}
$$

where $R_{1,1}=b+1$. In general, we can prove that

$$
\begin{aligned}
r_{2 n, 2 s}= & u^{-n-2 s} v^{-n-2 s} R_{0,2 s} a^{-n(n+1) / 2-2 s n-s(s+1)} \\
& \times b^{-n(n+1) / 2-2 s n-s^{2}} \prod_{i=1}^{2 s}\left(a^{i} b^{i}-1\right)^{-1}
\end{aligned}
$$

with $R_{0,2 s}=a^{s} b^{s} R_{0,2 s-1}+R_{1,2 s-1}$,

$$
\begin{aligned}
r_{2 n+1,2 s}= & u^{-n-2 s-1} v^{-n-2 s} R_{1,2 s} a^{-n(n+1) / 2-(2 s+1) n-(s+1)^{2}} \\
& \times b^{-n(n+1) / 2-2 s n-s(s+1)} \prod_{i=1}^{2 s}\left(a^{i} b^{i}-1\right)^{-1}
\end{aligned}
$$

with $R_{1,2 s}=R_{0,2 s-1}+a^{s} b^{s} R_{1,2 s-1}$ 


$$
\begin{aligned}
r_{2 n, 2 s+1}= & u^{-n-2 s-1} v^{-n-2 s-1} R_{0,2 s+1} a^{-n(n+1) / 2-(2 s+1) n-(s+1)^{2}} \\
& \times b^{-n(n+1) / 2-(2 s+1) n-s(s+1)} \prod_{i=1}^{2 s+1}\left(a^{i} b^{i}-1\right)^{-1}
\end{aligned}
$$

with $R_{0,2 s+1}=a^{s+1} b^{s} R_{0,2 s}+R_{1,2 s}$,

$$
\begin{aligned}
r_{2 n+1,2 s+1}= & u^{-n-2 s-2} v^{-n-2 s-1} R_{1,2 s+1} a^{-n(n+1) / 2-(2 s+2) n-(s+1)(s+2)} \\
& \times b^{-n(n+1) / 2-(2 s+1) n-(s+1)^{2}} \prod_{i=1}^{2 s+1}\left(a^{i} b^{i}-1\right)^{-1}
\end{aligned}
$$

with $R_{1,2 s+1}=R_{0,2 s}+a^{s} b^{s+1} R_{1,2 s}$.

We omit the detailed but routine induction.

Corollary 1.

$$
\left[0 ; \overline{u a^{k}, v a^{k}}\right]_{k=1}^{\infty}=\frac{\sum_{s=0}^{\infty} u^{-s-1} v^{-s} a^{-(s+1)(s+2) / 2} \prod_{i=1}^{s}\left(a^{i}-1\right)^{-1}}{\sum_{s=0}^{\infty} u^{-s} v^{-s} a^{-s(s+1) / 2} \prod_{i=1}^{s}\left(a^{i}-1\right)^{-1}},
$$

where $u$ and $v$ are rational so that ua and va are positive integers.

REMARK. If $u=v=1$, then we have the second relation of the main theorem in $[6]$.

Proof of Corollary 1. If $a=b$, then

$$
R_{0, s}=R_{1, s}=\prod_{i=1}^{s}\left(a^{i}+1\right) \quad(s=0,1, \ldots) .
$$

Thus, in Theorem 3,

$$
\begin{aligned}
& r_{0, s}=\sum_{s=0}^{\infty} u^{-s} v^{-s} a^{-s(s+1) / 2} \prod_{i=1}^{s}\left(a^{i}-1\right)^{-1} \\
& r_{1, s}=\sum_{s=0}^{\infty} u^{-s-1} v^{-s} a^{-(s+1)(s+2) / 2} \prod_{i=1}^{s}\left(a^{i}-1\right)^{-1}
\end{aligned}
$$

The theorem corresponding to Corollary 1 is the following. One can obtain it similarly by using the negative continued fraction expansion instead of the simple expansion. We omit the proof.

THEOREM 4. Let $a$ be an integer and $u$ a rational with $a>1$, ua, va $\in$ $\mathbb{Z}^{+}$and $v a \neq 2$. Then

$$
\begin{aligned}
{[0 ; u a-1,1, v a} & \left.-2, \overline{1, u a^{k+1}-2,1, v a^{k+1}-2}\right]_{k=1}^{\infty} \\
& =\frac{\sum_{s=0}^{\infty}(-1)^{s} u^{-s-1} v^{-s} a^{-(s+1)(s+2) / 2} \prod_{i=1}^{s}\left(a^{i}-1\right)^{-1}}{\sum_{s=0}^{\infty}(-1)^{s} u^{-s} v^{-s} a^{-s(s+1) / 2} \prod_{i=1}^{s}\left(a^{i}-1\right)^{-1}} .
\end{aligned}
$$


If $a=2$ and $v=1$, then

$$
\begin{aligned}
& {\left[0 ; 2 u-1,2, \overline{2^{k+1} u-2,1,2^{k+1}-2,1}\right]_{k=1}^{\infty}} \\
& =\frac{\sum_{s=0}^{\infty}(-1)^{s} u^{-s-1} 2^{-(s+1)(s+2) / 2} \prod_{i=1}^{s}\left(2^{i}-1\right)^{-1}}{\sum_{s=0}^{\infty}(-1)^{s} u^{-s} 2^{-s(s+1) / 2} \prod_{i=1}^{s}\left(2^{i}-1\right)^{-1}} .
\end{aligned}
$$

4. Hurwitzian continued fractions. We start from an extension of results of Elianu [2] and Lehmer [7].

\section{Theorem 5.}

$[0 ; u c, v(c+d), u(c+2 d), v(c+3 d), u(c+4 d), v(c+5 d), \ldots]$

$$
=\frac{\sum_{s=0}^{\infty}\left(s ! u^{s+1}(v d)^{s} \prod_{i=0}^{s}(c+i d)\right)^{-1}}{\sum_{s=0}^{\infty}\left(s !(u v d)^{s} \prod_{i=0}^{s-1}(c+i d)\right)^{-1}} .
$$

Proof. For simplicity put

$$
A_{n}=\left\{\begin{array}{ll}
u(c+n d) & \text { if } n \text { is even } \\
v(c+n d) & \text { if } n \text { is odd }
\end{array} \quad(n=0,1, \ldots) .\right.
$$

Consider the power series

$$
f_{n}(z)=r_{n, 0}+r_{n, 1} z+r_{n, 2} z^{2}+\ldots \quad(n=0,1, \ldots),
$$

satisfying

$$
f_{n}(z)=A_{n} f_{n+1}(z)+f_{n+2}(z)
$$

Then from

$$
\frac{f_{n}(z)}{f_{n+1}(z)}=A_{n}+\frac{z}{\frac{f_{n+1}(z)}{f_{n+2}(z)}}
$$

we obtain the desired continued fraction expansion

$$
\frac{f_{1}(1)}{f_{0}(1)}=[0 ; u c, v(c+d), u(c+2 d), v(c+3 d), \ldots] .
$$

We now determine $r_{n, 0}, r_{n, 1}, r_{n, 2}, \ldots$ in (3). First, compare the constant terms in (4) to get

$$
r_{n, 0}=A_{n} r_{n+1,0} \quad(n=0,1, \ldots),
$$

yielding $r_{0,0}=\prod_{j=0}^{n-1} A_{j} r_{n, 0}$. We can assume $r_{0,0}=1$ without loss of generality. Hence,

$$
r_{n, 0}=\prod_{j=0}^{n-1} A_{j}^{-1} \quad(n=0,1, \ldots) .
$$

Next, compare the coefficients of $z^{s}(s \geq 1)$ in $(2)$ to obtain

$$
r_{n, s}=A_{n} r_{n+1, s}+r_{n+2, s-1} \quad(n=0,1, \ldots),
$$


yielding

$$
r_{0, s}=\prod_{j=0}^{n-1} A_{j} r_{n, s}+\sum_{k=1}^{n} \prod_{j=0}^{k-2} A_{j} r_{k+1, s-1} .
$$

We shall prove by induction that

$$
r_{n, s}=\frac{1}{s !(u v d)^{s}} \prod_{i=0}^{s-1}(c+(n+i) d)^{-1} \prod_{j=0}^{n-1} A_{j}^{-1} .
$$

When $s=1$, by (6) and (5) we have

$$
\begin{aligned}
r_{0,1}= & \prod_{j=0}^{n-1} A_{j} r_{n, 1}+\sum_{k=1}^{n} \prod_{j=0}^{k-2} A_{j} \prod_{j=0}^{k} A_{j}^{-1} \\
= & \prod_{j=0}^{n-1} A_{j} r_{n, 1}+\frac{1}{u v d}\left(\left(\frac{1}{c}-\frac{1}{c+d}\right)+\left(\frac{1}{c+d}-\frac{1}{c+2 d}\right)\right. \\
& \left.\quad+\ldots+\left(\frac{1}{c+(n-1) d}-\frac{1}{c+n d}\right)\right) \\
= & \prod_{j=0}^{n-1} A_{j} r_{n, 1}+\frac{1}{u v d}\left(\frac{1}{c}-\frac{1}{c+n d}\right) .
\end{aligned}
$$

Separating the terms including $n$ from the constant terms without $n$, we obtain

$$
r_{n, 1}=\frac{1}{u v d(c+n d)} \prod_{j=0}^{n-1} A_{j}^{-1} .
$$

Assume that (7) is valid for $s-1$ and $s$. Then by (6) we have

$$
\begin{aligned}
r_{0, s+1}= & \prod_{j=0}^{n-1} A_{j} r_{n, s+1}+\sum_{k=1}^{n} \prod_{j=0}^{k-2} A_{j} \frac{1}{s !(u v d)^{s}} \prod_{i=0}^{s-1}(c+(k+i+1) d)^{-1} \prod_{j=0}^{k} A_{j}^{-1} \\
= & \prod_{j=0}^{n-1} A_{j} r_{n, s+1} \\
& +\frac{1}{s !(u v d)^{s}} \sum_{k=1}^{n} \frac{1}{u v(c+(k-1) d)(c+k d) \ldots(c+(k+s) d)} \\
= & \prod_{j=0}^{n-1} A_{j} r_{n, s+1}+\frac{1}{(s+1) !(u v d)^{s+1}} \\
& \times\left(\left(\frac{1}{c(c+d) \ldots(c+s d)}-\frac{1}{(c+d)(c+2 d) \ldots(c+(s+1) d)}\right)\right.
\end{aligned}
$$




$$
\begin{aligned}
& +\left(\frac{1}{(c+d)(c+2 d) \ldots(c+(s+1) d)}-\frac{1}{(c+2 d)(c+3 d) \ldots(c+(s+2) d)}\right) \\
& +\ldots+\left(\frac{1}{(c+(n-1) d)(c+n d) \ldots(c+(n+s-1) d)}\right. \\
& \left.\left.-\frac{1}{(c+n d)(c+(n+1) d) \ldots(c+(n+s) d)}\right)\right) \\
= & \prod_{j=0}^{n-1} A_{j} r_{n, s+1}+\frac{1}{(s+1) !(u v d)^{s+1}}\left(\frac{1}{c(c+d) \ldots(c+s d)}\right. \\
& \left.-\frac{1}{(c+n d)(c+(n+1) d) \ldots(c+(n+s) d)}\right) .
\end{aligned}
$$

Separating the terms including $n$ from the constant terms without $n$, we obtain

$$
r_{n, s+1}=\frac{1}{(s+1) !(u v d)^{s+1}} \prod_{i=0}^{s}(c+(n+i) d)^{-1} \prod_{j=0}^{n-1} A_{j}^{-1} .
$$

Thus, assertion (7) holds. Therefore,

$$
\begin{aligned}
& r_{0, s}=\frac{1}{s !(u v d)^{s}} \prod_{i=0}^{s-1}(c+i d)^{-1}, \\
& r_{1, s}=\frac{1}{s !(u v d)^{s}} \prod_{i=0}^{s-1}(c+(i+1) d)^{-1} A_{0}^{-1}=\frac{1}{s ! u^{s+1}(v d)^{s}} \prod_{i=0}^{s}(c+i d)^{-1} .
\end{aligned}
$$

EXAMPLE $1([2],[7])$.

$$
[0 ; \overline{c+k d}]_{k=1}^{\infty}=\frac{I_{c / d+1}\left(\frac{2}{d}\right)}{I_{c / d}\left(\frac{2}{d}\right)},
$$

where $I_{\lambda}(z)$ are the modified Bessel functions of the first kind, defined by

$$
I_{\lambda}(z)=\sum_{\nu=0}^{\infty} \frac{(z / 2)^{\lambda+2 \nu}}{\nu ! \Gamma(\lambda+\nu+1)} .
$$

Proof. Put $u=v=1$ and replace $c$ by $c+d$ in Theorem 5 . Then

$$
[0 ; \overline{c+k d}]_{k=1}^{\infty}=\frac{\sum_{s=0}^{\infty}\left(s ! d^{s} \prod_{i=1}^{s+1}(c+i d)\right)^{-1}}{\sum_{s=0}^{\infty}\left(s ! d^{s} \prod_{i=1}^{s}(c+i d)\right)^{-1}} .
$$

On the other hand, by the definition of the modified Bessel functions of the first kind,

$$
\begin{aligned}
I_{c / d}\left(\frac{2}{d}\right) & =\left(\frac{1}{d}\right)^{c / d} \sum_{s=0}^{\infty} \frac{1}{s ! d^{2 s} \Gamma(c / d+s+1)} \\
& =\frac{1}{d^{c / d} \Gamma(c / d+1)} \sum_{s=0}^{\infty} \frac{1}{s ! d^{s} \prod_{i=1}^{s}(c+i d)}
\end{aligned}
$$




$$
I_{c / d+1}\left(\frac{2}{d}\right)=\frac{1}{d^{c / d} \Gamma(c / d+1)} \sum_{s=0}^{\infty} \frac{1}{s ! d^{s} \prod_{i=1}^{s+1}(c+i d)} .
$$

EXAMPLE $2([14],(8))$.

$$
[0 ; \overline{(4 k-3) u,(4 k-1) v}]_{k=1}^{\infty}=\sqrt{\frac{v}{u}} \tanh \frac{1}{\sqrt{u v}} .
$$

Proof. Put $c=1$ and $d=2$ in Theorem 5. Then

$$
[0 ; \overline{(4 k-3) u,(4 k-1) v}]_{k=1}^{\infty}=\frac{\sum_{s=0}^{\infty}\left(u^{s+1} v^{s}(2 s+1) !\right)^{-1}}{\sum_{s=0}^{\infty}\left((u v)^{s}(2 s) !\right)^{-1}} .
$$

On the other hand, from

$$
\sinh x=\sum_{s=0}^{\infty} \frac{x^{2 s+1}}{(2 s+1) !} \quad \text { and } \quad \cosh x=\sum_{s=0}^{\infty} \frac{x^{2 s}}{(2 s) !},
$$

we have

$$
\sqrt{\frac{v}{u}} \tanh \frac{1}{\sqrt{u v}}=\sqrt{\frac{v}{u}} \cdot \frac{\sum_{s=0}^{\infty}(u v)^{-s-1 / 2}((2 s+1) !)^{-1}}{\sum_{s=0}^{\infty}(u v)^{-s}((2 s) !)^{-1}} .
$$

Theorem 6.

$$
[0 ; u c-1,1, v(c+d)-2,1, u(c+2 d)-2,1, v(c+3 d)-2,1, \ldots]
$$

$$
=\frac{\sum_{s=0}^{\infty}(-1)^{s}\left(s ! u^{s+1}(v d)^{s} \prod_{i=0}^{s}(c+i d)\right)^{-1}}{\sum_{s=0}^{\infty}(-1)^{s}\left(s !(u v d)^{s} \prod_{i=0}^{s-1}(c+i d)\right)^{-1}} .
$$

Proof. Consider the power series

$$
f_{n}(z)=r_{n, 0}+r_{n, 1} z+r_{n, 2} z^{2}+\ldots \quad(n=0,1, \ldots),
$$

satisfying

$$
f_{n}(z)=A_{n} f_{n+1}(z)-z f_{n+2}(z),
$$

where $A_{n}$ is the same as in the proof of Theorem 5 . Then

$$
\frac{f_{1}(z)}{f_{0}(z)}=\frac{z}{A_{1}-\frac{z}{A_{2}-\frac{z}{A_{3}-\ldots}}}
$$

entailing that

$$
\frac{f_{1}(1)}{f_{0}(1)}={ }^{-}\left[0 ; A_{1}, A_{2}, A_{3}, \ldots\right]
$$

By Lemma 1 we have

$$
\begin{aligned}
{[0 ; u c-1,1, v(c+d)-2,1} & , u(c+2 d)-2,1, v(c+3 d)-2,1, \ldots] \\
& =-[0 ; u c, v(c+d), u(c+2 d), v(c+3 d), \ldots] .
\end{aligned}
$$

The rest of the proof is similar to that of Theorem 5 . 
EXAMPLE $3([7])$.

$$
[0 ; c+d-1, \overline{1, c+(k+1) d-2}]_{k=1}^{\infty}=\frac{J_{c / d+1}\left(\frac{2}{d}\right)}{J_{c / d}\left(\frac{2}{d}\right)},
$$

where $J_{\lambda}(z)$ are the Bessel functions of the first kind, defined by

$$
J_{\lambda}(z)=\left(\frac{z}{2}\right)^{\lambda} \sum_{\nu=0}^{\infty} \frac{(i z / 2)^{2 \nu}}{\nu ! \Gamma(\lambda+\nu+1)} .
$$

Proof. Put $u=v=1$ and replace $c$ by $c+d$ in Theorem 6 . The result follows similarly to Example 1.

EXAMPLE 4.

$$
[0 ; u-1, \overline{1,(4 k-1) v-2,1,(4 k+1) u-2}]_{k=1}^{\infty}=\sqrt{\frac{v}{u}} \tan \frac{1}{\sqrt{u v}} .
$$

Proof. Put $c=1$ and $d=2$ in Theorem 6. Then

$$
\begin{aligned}
\sqrt{\frac{v}{u}} \tan \frac{1}{\sqrt{u v}} & =\sqrt{\frac{v}{u}} \cdot \frac{\sin (u v)^{-1 / 2}}{\cos (u v)^{-1 / 2}}=\frac{\sum_{s=0}^{\infty}(-1)^{s}\left(u^{s+1} v^{s}(2 s+1) !\right)^{-1}}{\sum_{s=0}^{\infty}(-1)^{s}\left(u^{s} v^{s}(2 s) !\right)^{-1}} \\
& ={ }^{-}[0 ; \overline{(4 k-3) u,(4 k-1) v}]_{k=1}^{\infty} \\
& =[0 ; u-1, \overline{1,(4 k-1) v-2,1,(4 k+1) u-2}]_{k=1}^{\infty} .
\end{aligned}
$$

REMARK. (1) If $u=a(>1)$ and $v=1$, then

$$
\frac{1}{\sqrt{a}} \tan \frac{1}{\sqrt{a}}=[0 ; a-1, \overline{1,4 k-3,1,(4 k+1) a-2}]_{k=1}^{\infty}
$$

as shown in $[14,(20)]$.

(2) If $u=v=a(>1)$, then

$$
\begin{aligned}
\tan \frac{1}{a} & =[0 ; a-1, \overline{1,(4 k-1) a-2,1,(4 k+1) a-2}]_{k=1}^{\infty} \\
& =[0 ; a-1, \overline{1,(2 k+1) a-2}]_{k=1}^{\infty}
\end{aligned}
$$

as shown in $[14,(17)]$.

(3) If $u=1$ and $v=a$ (including $a=1$ ), then

$$
\begin{aligned}
\sqrt{a} \tan \frac{1}{\sqrt{a}} & =[0 ; 0, \overline{1,(4 k-1) a-2,1,4 k-1}]_{k=1}^{\infty} \\
& =[1 ; \overline{(4 k-1) a-2,1,4 k-1,1}]_{k=1}^{\infty}
\end{aligned}
$$

as shown in $[14,(19)]$.

5. Some extended forms. Some Hurwitzian continued fraction expansions of tanh type (or tan type) cannot be derived directly from Theorem 5 (or Theorem 6, respectively). For example,

$$
2 \tan 1=[3 ; \overline{12 k-4,1,3 k-1,2,3 k, 1}]_{k=1}^{\infty}
$$


(Tasoev missed one 1 in [14, (21)]). But applying Raney's method [12], which is developed in [11], e.g., we can obtain it from $\tan 1=[1, \overline{2 k-1,1}]_{k=1}^{\infty}$. The continued fraction $\left[a_{0} ; a_{1}, a_{2}, \ldots\right]$ corresponds to the matrix product

$$
\left(\begin{array}{cc}
a_{0} & 1 \\
1 & 0
\end{array}\right)\left(\begin{array}{cc}
a_{1} & 1 \\
1 & 0
\end{array}\right)\left(\begin{array}{cc}
a_{2} & 1 \\
1 & 0
\end{array}\right) \ldots=R^{a_{0}} L^{a_{1}} R^{a_{2}} \ldots,
$$

where

$$
R^{a}=\left(\begin{array}{ll}
1 & a \\
0 & 1
\end{array}\right) \quad \text { and } \quad L^{a}=\left(\begin{array}{cc}
1 & 0 \\
a & 1
\end{array}\right) .
$$

Set

$$
A=\left(\begin{array}{cc}
c & 0 \\
0 & 1
\end{array}\right) \text { and } A^{\prime}=\left(\begin{array}{ll}
1 & 0 \\
0 & c
\end{array}\right) \text {. }
$$

Using the transition formulae

$$
\begin{aligned}
A R & =R^{c} A, & A^{\prime} L & =L^{c} A^{\prime}, \\
A L^{c} & =L A, & A^{\prime} R^{c} & =R A^{\prime}, \\
A L R^{c-1} & =R^{c-1} L A^{\prime}, & A^{\prime} R L^{c-1} & =L^{c-1} R A
\end{aligned}
$$

in the case where $c=2$, we have, for $k=1,2, \ldots$,

$$
\begin{aligned}
A^{\prime} L^{6 k-3} R L^{6 k-1} R L^{6 k+1} R & =L^{12 k-6} A^{\prime} R L^{6 k-1} R L^{6 k+1} R \\
& =L^{12 k-6} L R A L^{6 k-2} R L^{6 k+1} R \\
& =L^{12 k-5} R L^{3 k-1} R^{2} A L^{6 k+1} R \\
& =L^{12 k-5} R L^{3 k-1} R^{2} L^{3 k} R L A^{\prime} .
\end{aligned}
$$

Therefore, by the correspondence of continued fractions with matrix products, we have

$2 \tan 1=2[1 ; 1,1,3,1,5,1,7,1,9,1, \ldots] \longleftrightarrow$

$$
\begin{aligned}
A R L R L^{3} R L^{5} R L^{7} R L^{9} \ldots & =R^{2} R L A^{\prime} L^{3} R L^{5} R L^{7} R L^{9} R L^{11} R L^{13} R L^{15} \ldots \\
& =R^{3} L L^{7} R L^{2} R^{2} L^{3} R L L^{19} R L^{5} R^{2} L^{6} R L L^{31} \ldots \\
& \longleftrightarrow[3 ; 8,1,2,2,3,1,20,1,5,2,6,32, \ldots] .
\end{aligned}
$$

In a similar manner one can obtain the following extended forms.

Proposition 1.

$$
\begin{aligned}
& 2 \sqrt{\frac{v}{u}} \tanh \frac{1}{\sqrt{u v}} \\
& =\left\{\begin{array}{lr}
{\left[0 ; \overline{(4 k-3) \frac{u}{2},(8 k-2) v}\right]_{k=1}^{\infty}} & \text { if } u \text { is even; } \\
{\left[0 ; \overline{4 k u-\frac{7 u+1}{2}, 1,1,4 k v-\frac{5}{2} v-1,1,1,}\right.} & \text { if } u \text { is odd and } v \text { is even; } \\
\frac{\left[0 ; \overline{6 k u-\frac{3 u+1}{2},(16 k-2) v}\right]_{k=1}^{\infty}}{\frac{11 u+1}{2}, 1,1,6 k v-\frac{9 v+1}{2},(24 k-14) u, 6 k v-\frac{5 v+1}{2},} \\
\left.\frac{1,1,6 k u-\frac{3 u+1}{2},(24 k-2) v}{\infty}\right]_{k=1}^{\infty} & \text { if } u \text { is odd and } v \text { is odd. }
\end{array}\right.
\end{aligned}
$$




$$
\begin{aligned}
& \frac{1}{2} \sqrt{\frac{v}{u}} \tanh \frac{1}{\sqrt{u v}} \\
& =\left\{\begin{array}{l}
{\left[0 ; \overline{(8 k-6) u,(4 k-1) \frac{v}{2}}\right]_{k=1}^{\infty}} \\
{\left[0 ; \overline{(16 k-14) u, 4 k v-\frac{5 v+1}{2}, 1,1,4 k u-\frac{3}{2} u-1,}\right.} \\
\frac{\left.1,1,4 k v-\frac{v+1}{2}\right]_{k=1}^{\infty}}{\left[0 ;(24 k-22) u, 6 k v-\frac{9 v+1}{2}, 1,1,6 k u-\frac{7 u+1}{2},(24 k-10) v,\right.}
\end{array} \text { if } v \text { is even and } v\right. \text { is odd; } \\
& \left.\overline{6 k u-\frac{3 u+1}{2}, 1,1,6 k v-\frac{v+1}{2}}\right]_{k=1}^{\infty} \\
& \text { if } u \text { is odd and } v \text { is odd. }
\end{aligned}
$$

Proposition 2.

$$
\begin{aligned}
& 2 \sqrt{\frac{v}{u}} \tan \frac{1}{\sqrt{u v}} \\
& =\left\{\begin{array}{l}
{\left[0 ; \frac{u}{2}-1, \overline{1,8 k v-2 v-2,1,2 k u+\frac{u}{2}-2}\right]_{k=1}^{\infty}} \\
{\left[0 ; \frac{u-1}{2}, \overline{2,4 k v-\frac{5}{2} v-1,2,4 k u-\frac{3 u+3}{2}, 1},\right.} \\
{\left[0 ; \frac{u-1}{2}, \overline{2,6 k v-\frac{9 v+3}{2}, 1,24 k u-14 u-2,1,6 k v-\frac{5 v+3}{2}, 2,6 k u-\frac{3 u+3}{2},}\right.} \\
\left.\frac{1,24 k v-2 v-2,1,6 k u+\frac{u-3}{2}}{16 k-2,1,4 k u+\frac{u-3}{2}}\right]_{k=1}^{\infty}
\end{array} \text { if } u\right. \text { is even; }
\end{aligned}
$$

$\frac{1}{2} \sqrt{\frac{v}{u}} \tan \frac{1}{\sqrt{u v}}$

$$
=\left\{\begin{array}{cc}
{\left[0 ; 2 u-1, \overline{1,2 k v-\frac{v}{2}-2,1,8 k u+2(u-1)}\right]_{k=1}^{\infty}} & \text { if } v \text { is even; } \\
{\left[0 ; 2 u-1, \overline{1,4 k v-\frac{5 v+3}{2}, 2,4 k u-\frac{3}{2} u-1,2,4 k v-\frac{v+3}{2}, 1,}\right.} & \text { if } u \text { is even and } v \text { is odd } ; \\
\left.\frac{16 k u+2(u-1)}{16}\right]_{k=1}^{\infty} & \\
\left.\frac{6 k u-\frac{3 u+3}{2}, 2,6 k v-\frac{v+3}{2}, 1,24 k u+2(u-1)}{6}\right]_{k=1}^{\infty} & \text { if both } u \text { and } v \text { are odd. }
\end{array}\right.
$$

Note that the negative continued fraction expansion does not completely correspond to the simple continued fraction expansion unless $u$ is even in the case multiplied by 2 or $v$ is even in the case divided by 2 . One of the reasons is that the partial quotients in the negative continued fraction expansion do not allow the integer 1 , but the integers larger than 1 . Here is the corresponding relation:

$$
{ }^{-}[\ldots, a+1,2, b+1, \ldots] \longleftrightarrow[\ldots, a, 1,1, b, \ldots] .
$$


The cases multiplied or divided by $3,4,5, \ldots$ may be obtained but are not simple. For instance,

$$
\begin{aligned}
\frac{1}{3} \tan 1 & =[0 ; 1,1,12, \overline{1,4 k-3,3,4 k-2,1,1,1}, \\
& ={ }^{-}[0 ; 2,14, \overline{4 k-2,1,36 k+1,1,4 k-1,1,36 k+13}]_{k=1}^{\infty}
\end{aligned}
$$

and

$$
\begin{aligned}
& \frac{1}{3} \tanh 1 \\
& \quad=[0 ; \overline{36 k-33,4 k-3,36 k-21,4 k-2,2,1,4 k-2,2,1,4 k-1}]_{k=1}^{\infty} .
\end{aligned}
$$

Acknowledgements. The research for this paper was initiated when the author was visiting the Mathematisches Institut der Universität zu Köln. He thanks the Institut for its warm hospitality. The author is grateful to Professor Dr. P. Bundschuh for many fruitful discussions. He also thanks the referee for several useful suggestions.

\section{References}

[1] C. S. Davis, On some simple continued fractions connected with e, J. London Math. Soc. 20 (1945), 194-198.

[2] J. Elianu, Sur le nombre $[\alpha, \alpha+\beta, \alpha+2 \beta, \ldots, \alpha+n \beta, \ldots]$, Rev. Roumaine Math. Pures Appl. 20 (1975), 1061-1071.

[3] A. Hurwitz, Über eine besondere Art der Kettenbruch-Entwicklung reeller Grössen, Acta Math. 12 (1889), 367-405.

[4] -, Über die Kettenbruch-Entwicklung der Zahl e, Schriften der physikalisch-ökonomischen Gesellschaft zu Königsberg in Pr. 32 (1891), 59-62; also in: Mathematische Werke von Adolf Hurwitz, Band II, Birkhäuser, Basel, 1933.

[5] —, Über die Kettenbrüche, deren Teilnenner arithmetische Reihen bilden, Vierteljahrsschrift der Naturforschenden Gesellschaft in Zürich 41 (1896), 34-64; also in: Mathematische Werke von Adolf Hurwitz, Band II, Birkhäuser, Basel, 1933.

[6] T. Komatsu, On Tasoev's continued fractions, Math. Proc. Cambridge Philos. Soc. 134 (2003), to appear.

[7] D. H. Lehmer, Continued fractions containing arithmetic progressions, Scripta Math. 29 (1973), 17-24.

[8] K. R. Matthews and R. F. C. Walters, Some properties of the continued fraction expansion of $(m / n) e^{1 / q}$, Proc. Cambridge Philos. Soc. 67 (1970), 67-74.

[9] O. Perron, Die Lehre von den Kettenbrüchen, Chelsea reprint of the 1929 edition (§29-§32), 1950, 126-138.

[10] C. G. Pinner, More on inhomogeneous Diophantine approximation, J. Théor. Nombres Bordeaux 13 (2001), 539-557.

[11] A. J. van der Poorten, Continued fraction expansions of values of the exponential function and related fun with continued fractions, Nieuw Arch. Wisk. 14 (1996), 221-230. 
[12] G. Raney, On continued fractions and automata, Math. Ann. 206 (1973), 265-283.

[13] B. G. Tasoev, Certain problems in the theory of continued fractions, Trudy Tbiliss. Univ. Mat. Mekh. Astronom. 16/17 (1984), 53-83 (in Russian).

[14] —, Rational approximations to certain numbers, Mat. Zametki 67 (2000), 931-937 (in Russian); English transl.: Math. Notes 67 (2000), 786-791.

[15] D. S. Thakur, Patterns of continued fractions for the analogues of $e$ and related numbers in the function field case, J. Number Theory 66 (1997), 129-147.

[16] H. S. Wall, Analytic Theory of Continued Fractions, D. van Nostrand Company, Toronto, 1948.

Faculty of Education

Mie University

Mie, 514-8507, Japan

E-mail: komatsu@edu.mie-u.ac.jp

Received on 30.10.2001

and in revised form on 21.5.2002 